Proceedings of the International School and Conference on Optics and Optical Materials, ISCOM07, Belgrade, Serbia, September 3-7, 2007

\title{
Laser- and Light-Induced Autofluorescence Spectroscopy of Human Skin in Dependence on Excitation Wavelengths
}

\begin{abstract}
I. Bliznakova*, E. Borisova And L. Avramov
Institute of Electronics, Bulgarian Academy of Sciences, Sofia, Bulgaria

Human skin contains various types of native fluorophores and absorbers with unique absorption and emission spectra, different quantum efficiency, concentration and spatial distribution within the skin. Autofluorescence spectroscopy is applied as diagnostic tool for cutaneous tumor detection that increases the importance of evaluation of natural existing fluorophores and unification of data for given class of pathologies. In the current study, several excitation sources in the region 337-405 $\mathrm{nm}$ are applied, to achieve information about typical autofluorescent properties of normal human skin.
\end{abstract}

PACS numbers: 42.72.Bj, 87.15.M-, 87.64.kv

\section{Introduction}

Optical spectroscopic techniques importance in the clinical practice is raised rapidly in the last two decades. With increase in knowledge about optical properties of different tissues and their pathologies, as well as with the improvement of spectroscopic equipment and the optimization of measurement methodologies new diagnostic approaches are introduced for the medical diagnosis purposes [1-4].

Biological tissues contain chromophores that absorb light, as well as fluorophores that absorb and reemit (fluoresce) light. Light absorption depends on the chromophores' content and their distribution within the organic matter. Biotissues are also structurally heterogeneous that promote strong light scattering $[1,2]$. The diverse optical properties of biological tissues make their analysis quite complicate, but also allow achieving of highly sensitive and rapid spectroscopic methods for detection of small changes in their healthy status.

${ }^{*}$ corresponding author; e-mail: irbliznakova@abv.bg 
Autofluorescence (AF) is one of the most sensitive spectroscopic techniques and it is used for diagnosis and follow-up of dermatological diseases, such as erythrasma and acne [5]. This method is also applied for skin cancer detection and lesion type determination $[1,6,7]$.

In the current work there are presented some comparative results of using of $\mathrm{N}_{2}$ laser $(\lambda=337.1 \mathrm{~nm}$ ) and light emitting diodes (LEDs) (with emission in $365,375,385$, and $405 \mathrm{~nm}$ ) as excitation sources for cutaneous fluorescence investigations and the evaluation of autofluorescence properties of normal human skin in vivo.

\section{Materials and methods}

\subsection{Fluorescent measurements}

For these experiments, the fluorescence is stimulated by $\mathrm{N}_{2}$ laser $(\lambda=$ $337.1 \mathrm{~nm}$ ) and LEDs with maximum emission at 365, 375, 385, and $400 \mathrm{~nm}$. The signal is transmitted from the source of light to the investigated objects and from the investigated objects to the detector system by quartz optical fibers. The laser applied uses longitudinal pumping ( $\mathrm{N}_{2}$ laser, ILGI-503, Russia). The technical characteristics of the light diodes used in this experiment are: UVLED365-10 at $\lambda=365 \mathrm{~nm}, P=1.4 \mathrm{~mW}, \lambda=10 \mathrm{~nm}$; UVLED380-10 with $\lambda=380 \mathrm{~nm}, P=3.4 \mathrm{~mW}, \lambda=10 \mathrm{~nm}$ ("Roithner-Laser Technik" GmbH, Austria); UV LED L4-1-U5TH15-1 (LED Supply Ltd., USA) with $\lambda=395 \mathrm{~nm}$, $P=10 \mathrm{~mW}, \lambda=15 \mathrm{~nm}$; OTLH-0360-UV-400 (Rhopoint Components Ltd, UK) with $\lambda=405 \mathrm{~nm}, P=120 \mathrm{~mW}, \lambda=15 \mathrm{~nm}$. In Fig. 1 there is presented schematically view of the experimental setup.

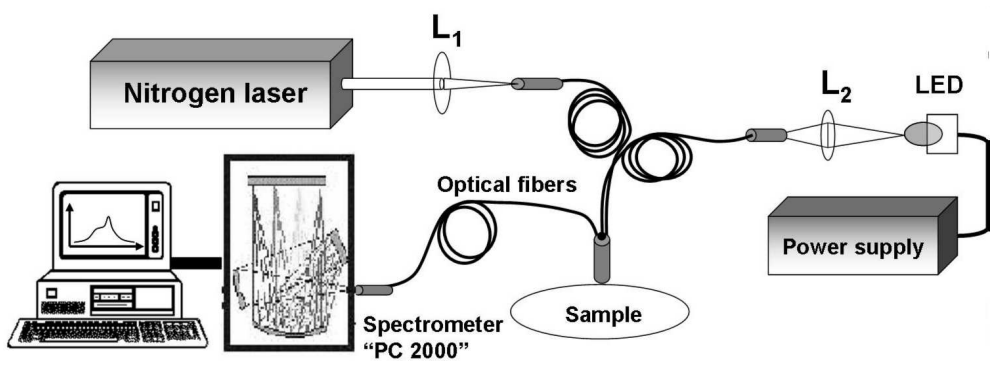

Fig. 1. Schematic view of the experimental setup used for measurements of fluorescent properties of human skin in vivo, using different excitation sources.

\subsection{Skin samples}

Healthy volunteers, representative of skin phototypes typical of Bulgarian region, namely phototypes I, II, and III were investigated on several anatomic sites: palm, medial, and lateral part of the forearm, using different excitation sources in the region $337-405 \mathrm{~nm}$. 


\section{Results and discussion}

The spectra, presented in this work, are smoothed and averaged in dependence of anatomic origin and skin phototype. Every autofluorescence spectrum detected in vivo is a superposition of fluorescence spectra of endogenous chromophores existing in the tissue under investigation distorted by re-absorption of tissue pigments, mainly blood and melanin. The spectral shape of normal skin fluorescence has no significant differences between volunteers, for a given excitation wavelength. Intensity changes are more pronounced due to different skin phototype and anatomic area, as in both cases different level of melanin and hemoglobin pigmentation could be observed. Detected slight differences in spectral shape are only for the case of palm skin fluorescence spectra versus other anatomic sites, where lack of melanin leads to deeper penetration of excitation and respectively for emission light. In this case influence of hemoglobin re-absorption of the fluorescence from deeper dermal layer is well pronounced. Strong changes are observed in the fluorescence spectra detected when using different excitation wavelengths as different fluorophores are included in the autofluorescence response of the tissue investigated.

\subsection{Results - repeatability}

To investigate repeatability of the measurements and possible differences from patient to patient, a comparison of the fluorescence spectra from several volunteers from one skin phototype and from the same anatomic area is carried out. The spectral shape observed is similar for all cases, but some slight differences in maximal intensity, related to pigmentation and blood content deviations are pronounced. Due to the same slight absorber concentrations differences from patient to patient differences in the spectral shape are observed.
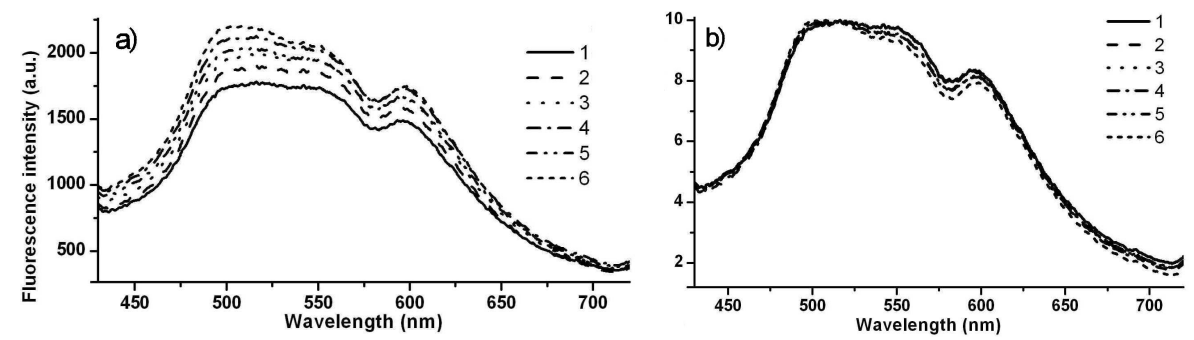

Fig. 2. Comparison of the fluorescence spectra obtained from lateral part of the forearm of six volunteers with phototype I, excitation used at $385 \mathrm{~nm}$, unnormalized with respect to maximum (left) and normalized with respect to maximum (right).

However, these differences are relatively weakly pronounced and do not change general conclusions about good repeatability of the fluorescence spectra shape between patients, with additional condition about the same skin phototype and anatomic area investigated, as these two parameters have strong influence on 
the spectra detected. The comparison of the fluorescence spectra from the lateral part of the forearm between the two curves shows a good repeatability of the received data in Fig. 2.

\subsection{Influence of excitation wavelength}

In comparison of fluorescence spectra using different excitation wavelengths there is clearly observed the appearance of new emission maxima and changes in fluorescence intensity, depending on absorption for given excitation wavelength of exact fluorophore. In Fig. 2 there are presented fluorescence spectra, normalized with respect to maximum for several excitation wavelengths. The normalization is applied to reveal better the spectral shape changes occurred.

With the increase in the excitation wavelength, new fluorophores are involved in the shape formation of fluorescence spectrum. This is related to deeper penetration of long-wavelength excitation [6], to higher absorption at given wavelength for some additional fluorophores and to differences in pigments influence, especially hemoglobin - see the minima detected at 545 and $575 \mathrm{~nm}$ in Fig. 3 [8, 9].

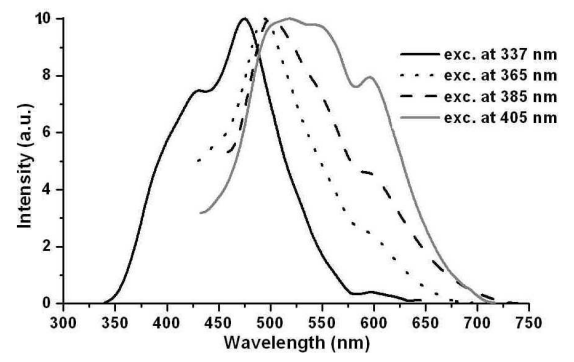

Fig. 3. Normalized with respect to maximum fluorescence spectra of normal human skin from one volunteer at different excitation wavelengths applied.

\subsection{Influence of anatomic area}

In the fluorescence spectra obtained several typical spectral features were observed, related to fluorescence of some endogenous chromophores on one side and to re-absorbance of the fluorescence by typical skin pigments on the other side.

These features are the maxima at the region of 470-500 nm, resulting from collagen and elastin cross-links fluorescence, maximally at the region 440-470 nm, related to NADH and FAD, which are main co-enzymes in cutaneous tissues.

The fluorescence peak at $550-600 \mathrm{~nm}$ is related to keratin and maximum obtained with excitation at $605 \mathrm{~nm}$, which lies at the $800-830 \mathrm{~nm}$ is related to some lipids in the human skin. Minima in the fluorescence spectra, related to re-absorption from the cutaneous pigment - hemoglobin are observed at $420 \mathrm{~nm}$ and $540-575 \mathrm{~nm}$. General decrease in the signals depending from skin phototype and anatomic area melanin pigmentation is also observed during measurements, 

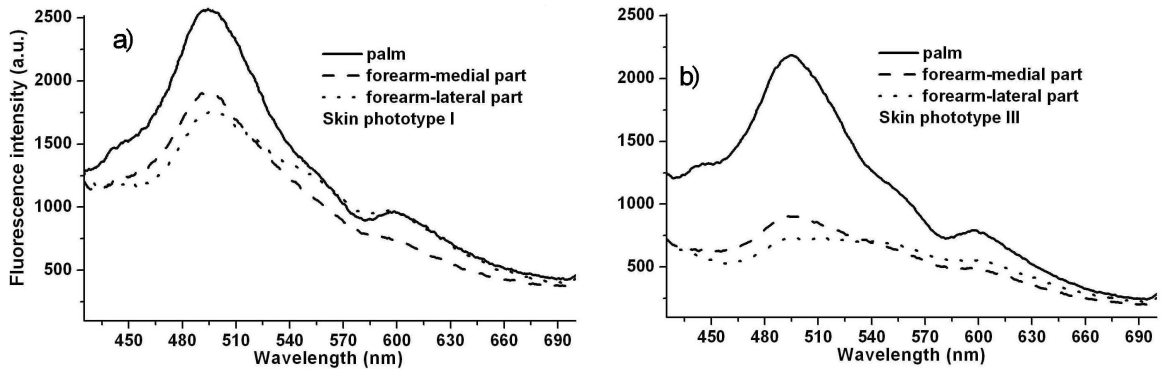

Fig. 4. Comparison of fluorescence spectra from different anatomic areas for phototype I (left) and phototype III (right), using excitation $365 \mathrm{~nm}$.

Fig. 4. This decrease is related to non-selective absorption spectrum of the melanin pigment, which is characterized with slow rise from red to blue spectral region $[1,2,10,11]$.

TABLE

Fluorescent compounds and structures, typical of human skin with their absorption and emission maxima.

\begin{tabular}{l|c|c|c}
\hline \hline Skin chromophore & Description & $\begin{array}{c}\text { Absorption } \\
\text { maxima [nm] }\end{array}$ & $\begin{array}{c}\text { Fluorescence } \\
\text { maxima [nm] }\end{array}$ \\
\hline Collagen & structural protein & 325 & 400,405 \\
Collagen cross-links & structural proteins & 370 & $460-490$ \\
Elastin & structural protein & 290,325 & 340,400 \\
Elastin cross-links & structural proteins & $420-460$ & $500-540$ \\
Keratin & structural protein & 370 & $460-500$ \\
FAD, flavins & co-enzymes & 450 & 535 \\
NADH & co-enzyme & 290,350 & 440,460 \\
Phospholipids & lipids & $340-440$ & $430-460,540-560$
\end{tabular}

In Table there are classified the fluorophores with main contribution to the normal and abnormal skin fluorescence spectra with their absorption and emission maxima, respectively.

\section{Conclusions}

With the increase in the excitation wavelength, new fluorophores are involved in the shape formation of fluorescence spectrum. This is related to deeper penetration of long-wavelength excitation, to higher absorption at given wavelength for some additional fluorophores and to differences in pigments influence.

Optimization of fluorescence detection could give ones broad opportunities to increase the sensitivity and specificity for early diagnosis and differentiation of 
cutaneous lesions. Application of cheaper light sources as LEDs could also help to spread the optical diagnostic methods in clinical practice.

\section{Acknowledgments}

This work was supported by the Bulgarian Ministry of Education and Science under grant MUF-03/05 "Development of apparatus and methods for optical biopsy of human skin".

\section{References}

[1] S. Svanberg, Phys. Scr. T 110, 39 (2004).

[2] J. Bigio, J.R. Mourant, Phys. Med. Biol. 42, 803 (1997).

[3] L. Bachmann, D. Zezell, A. da Costa Ribeiro, L. Gomes, A. Ito, Appl. Spectr. Rev. 41, 575 (2006).

[4] L. Avramov, E. Borisova, P. Townsend, L. Valberg, Mater. Sci. Forum 480-481, $411(2005)$.

[5] H. Ahn, S. Kim, Y. Kye, Skin Res. Tech. 12, 289 (2006).

[6] A.R. Young, Phys. Med. Biol. 42, 789 (1997).

[7] E. Borisova, P. Troyanova, L. Avramov, Proc. SPIE 6257, 0 U1 (2006).

[8] P. Troyanova, E. Borisova, V. Stoyanova, L. Avramov, Proc. SPIE 6284, 0K1 (2006).

[9] N. Kollias, G. Zonios, G. Stamatas, Vibrat. Spectrosc. 28, 17 (2002).

[10] Yu. Sinichkin, S. Utz, A. Mavliutov, H. Pilipenko, J. Biomed. Opt. 3, 201 (1998).

[11] R. Na, I. Stender, L. Ma, H. Wulf, Skin Res. Tech. 6, 112 (2000). 